\title{
Molecular Dynamics in Chlorine Compounds Using X-Ray Thermal Parameters and Nuclear Quadrupole Resonance Data
}

\author{
L. Ramu*, R. Banu, S. Rani and R. Chandramani \\ Department of Physics, Bangalore University, Bangalore-560056, India
}

(Received February 20, 2006)

\begin{abstract}
Molecular dynamics (torsional frequencies) in several chlorine compounds were evaluated on the basis of X-ray thermal parameter data and nuclear quadrupole resonance data using Bayer's and Brown's approximation. It was found that the values obtained in both cases are in good agreement. The above approach is a good illustration of the supplementary nature of the data from X-ray studies in relation to nuclear quadrupole resonance studies of compounds in solid state.
\end{abstract}

PACS numbers: $76.60 . \mathrm{Gv}$

\section{Introduction}

Study of nuclear quadrupole resonance (NQR) in molecular solids gives useful information about the molecular dynamics (torsional frequencies) and phase transitions in solids [1]. Usually the torsional frequencies, namely $f_{x}$ and $f_{y}$, are calculated using NQR frequency and crystal structure data, by numerical analysis [2]. The values will be compared with Fourier transformed IR/IR data.

Here an approach has been made to evaluate torsional frequencies at room temperature in number of chlorine compounds using crystal structure and the available X-ray thermal parameters. In order to evaluate the torsional frequencies the mean square angular displacements of various $\mathrm{C}-\mathrm{Cl}$ bond directions and the available X-ray thermal parameter has been utilized.

\section{Method of calculation}

The temperature factor of an atom for any set of lattice planes $(h k l)$ depends on the interplanar spacing and on the magnitude of vibration perpendicular to the

*corresponding author; e-mail: ramukls@yahoo.com 
planes. The expression for general temperature factor is given as

$$
\begin{aligned}
T= & \exp \left[-2 \pi^{2}\left(U_{11} h^{2} a^{* 2}+U_{22} k^{2} b^{* 2}+U_{33} l^{2} c^{* 2}+2 U_{12} h k a^{*} b^{*}\right.\right. \\
& \left.\left.+2 U_{13} h l a^{*} b^{*}+2 U_{23} h l b^{*} c^{*}\right)\right],
\end{aligned}
$$

where $a^{*}, b^{*}$, and $c^{*}$ are the reciprocal lattice parameters and $U_{i j}$ are called thermal parameters expressed in terms of mean square amplitudes of vibration in $\mathrm{pm}^{2}$. The off-diagonal thermal parameters have been neglected, since they are relatively small compared to the diagonal terms. The mean square angular displacement $\left\langle\theta^{2}\right\rangle$ is evaluated using the thermal parameters in the following manner.

TABLE I

Mean square atomic displacements of carbon and chlorine atoms in certain chlorine

\begin{tabular}{|c|c|c|c|c|c|c|}
\hline Sl. No. & Compound & Atom & $\begin{array}{c}U_{11} \\
{\left[\mathrm{pm}^{2}\right]}\end{array}$ & $\begin{array}{c}U_{22} \\
{\left[\mathrm{pm}^{2}\right]}\end{array}$ & $\begin{array}{c}U_{33} \\
{\left[\mathrm{pm}^{2}\right]}\end{array}$ & $\begin{array}{c}\langle U\rangle \\
{\left[\mathrm{pm}^{2}\right]}\end{array}$ \\
\hline \multirow[t]{4}{*}{1} & 2,5-dichlorophenol & $\mathrm{Cl}(1)$ & 23 & 38 & 05 & 22.000 \\
\hline & line I & $\mathrm{C}(4)$ & 17 & 28 & 02 & 15.666 \\
\hline & & $\mathrm{Cl}(2)$ & 23 & 37 & 03 & 21.000 \\
\hline & line II & $\mathrm{C}(7)$ & 17 & 27 & 03 & 15.666 \\
\hline \multirow[t]{4}{*}{2} & 2,6-dichlorophenol & $\mathrm{C}(4)$ & 74 & 60 & 316 & 150.00 \\
\hline & line I & $\mathrm{Cl}(1)$ & 94 & 71 & 416 & 193.66 \\
\hline & & $\mathrm{C}(8)$ & 58 & 62 & 381 & 165.66 \\
\hline & line II & $\mathrm{Cl}(2)$ & 64 & 100 & 468 & 210.66 \\
\hline \multirow[t]{4}{*}{3} & 3,5-dichlorophenol & $\mathrm{C}(5)$ & 62 & 635 & 54 & 250.33 \\
\hline & line I & $\mathrm{Cl}(1)$ & 82 & 1010 & 64 & 385.33 \\
\hline & & $\mathrm{C}(7)$ & 68 & 676 & 39 & 261.00 \\
\hline & line II & $\mathrm{Cl}(2)$ & 88 & 1005 & 46 & 379.66 \\
\hline \multirow[t]{2}{*}{4} & 2,5-dichloro-3,6-dihydroxy & $\mathrm{C}$ & 222 & 265 & 153 & 213.33 \\
\hline & $p$-benzoquinine & $\mathrm{Cl}$ & 271 & 320 & 166 & 252.33 \\
\hline \multirow[t]{6}{*}{5} & aniline-2,4,5-trichlorophenol & $\mathrm{C}(8)$ & 111 & 115 & 27 & 80.333 \\
\hline & & $\mathrm{Cl}(1)$ & 166 & 155 & 37 & 119.66 \\
\hline & line I & $\mathrm{C}(10)$ & 183 & 92 & 42 & 105.66 \\
\hline & line II & $\mathrm{Cl}(2)$ & 211 & 115 & 43 & 123.00 \\
\hline & line III & $\mathrm{C}(11)$ & 122 & 133 & 29 & 94.666 \\
\hline & & $\mathrm{Cl}(3)$ & 172 & 181 & 37 & 130.00 \\
\hline \multirow[t]{2}{*}{6} & 2-amino-5-chloropyridine & $\mathrm{C}(5)$ & 360 & 2101 & 1424 & 1295.0 \\
\hline & & $\mathrm{Cl}(1)$ & 391 & 2930 & 2412 & 1911.0 \\
\hline \multirow[t]{2}{*}{7} & monochloro acetic acid & $\mathrm{C}(2)$ & 479 & 662 & 414 & 518.33 \\
\hline & & $\mathrm{Cl}(1)$ & 407 & 522 & 378 & 435.67 \\
\hline
\end{tabular}
compounds. 
Let us suppose that the mean square displacements of two atoms forming a bond are given by $\left\langle U_{1}^{2}\right\rangle$ and $\left\langle U_{2}^{2}\right\rangle$ and the length of the bond is $l_{12}$, then the mean square displacements of the bond direction would be approximately given by

$$
\left\langle\theta^{2}\right\rangle=\left(\left\langle U_{1}^{2}\right\rangle-\left\langle U_{2}^{2}\right\rangle\right) / l_{12}
$$

The mean square angular displacement values calculated using the above equation are given in Table I.

The NQR motional averaging has been shown to arise from temperature dependent tilting of the $z$-axis of the electric field gradient (EFG) tensor. One can therefore estimate $\left\langle\theta^{2}\right\rangle$ for various $\mathrm{C}-\mathrm{Cl}$ bond directions with the respective $\mathrm{EFG}$ $z$-directions through the NQR data. The torsional frequencies of the molecules are evaluated using the formula

$$
\nu_{\mathrm{T}}=\nu_{0}\left\{1-\frac{3 h}{8 \pi^{2}} \frac{1}{A f_{\mathrm{T}}^{2}}\left[\frac{1}{2}+\frac{1}{\exp \left(h f_{\mathrm{T}} / k T-1\right)}\right]\right\} .
$$

Using high temperature approximation $[3,4], h f_{\mathrm{T}} / k T \ll 1$, Eq. (3) can be written as

$$
\nu_{\mathrm{T}}=\nu_{0}\left(1-3 k T / 8 \pi^{2} A c^{2} f_{\mathrm{T}}^{2}\right)
$$

TABLE II

Parameters used for torsional frequency calculations ( $A$ is moment of inertia).

\begin{tabular}{c|c|c|c|c|c}
\hline \hline $\begin{array}{c}\text { Sl. } \\
\text { No. }\end{array}$ & Compound & $\begin{array}{c}A_{x} \\
{\left[10^{-40}\right.} \\
\left.\mathrm{g} \mathrm{cm}^{2}\right]\end{array}$ & $\begin{array}{c}A_{y} \\
{\left[10^{-40}\right.} \\
\left.\mathrm{g} \mathrm{cm}^{2}\right]\end{array}$ & $A=\left(A_{x}+A_{y}\right) / 2$ \\
\hline 1 & 2,5 -dichlorophenol & & $\left.\mathrm{g} \mathrm{cm}^{2}\right]$ \\
\hline 2 & $\begin{array}{c}\text { line I } \\
\text { line II }\end{array}$ & $\mathrm{Cl}(1)$ & 478.721 & 351.688 & 415.207 \\
& 2,6 -dichlorophenol & & & & 415.207 \\
\hline 3 & line I & $\mathrm{Cl}(1)$ & 1283.95 & 753.556 & 1018.75 \\
& line II & $\mathrm{Cl}(2)$ & 1306.08 & 787.538 & 1046.81 \\
\hline 4 & line I & $\mathrm{Cl}(1)$ & 1505.0 & 863.897 & 1184.44 \\
& line II & $\mathrm{Cl}(2)$ & 1515.0 & 863.897 & 1189.44 \\
\hline 5 & aniline-2,4,5-trichlorophenol & & & & \\
& line I & $\mathrm{Cl}(1)$ & 2201.27 & 5119.23 & 3660.23 \\
& line II & $\mathrm{Cl}(2)$ & 2201.27 & 5119.23 & 3660.23 \\
& line III & $\mathrm{Cl}(3)$ & 2178.39 & 5335.97 & 3757.18 \\
\hline 6 & 2-amino-5-chloropyridine & $\mathrm{Cl}(1)$ & 978.960 & 839.930 & 909.445 \\
\hline 7 & monochloro acetic acid & $\mathrm{Cl}(1)$ & 11918.3 & 5755.07 & 8836.50 \\
\hline
\end{tabular}


Equation (4) can be expressed in terms of mean square angular displacement as

$$
\nu_{\mathrm{T}}=\nu_{0}\left[1-(3 / 2)\left\langle\theta^{2}\right\rangle\right]
$$

Comparing Eq. (4) and Eq. (5), the mean square angular displacement $\left\langle\theta^{2}\right\rangle$ is given by

$$
\left\langle\theta^{2}\right\rangle=k T / 4 \pi^{2} c^{2} A f_{\mathrm{T}}^{2}
$$

Therefore

$$
f_{\mathrm{T}}=\left[k T / 4 \pi^{2} c^{2} A\left\langle\theta^{2}\right\rangle\right]^{1 / 2}
$$

where $A=\left(A_{x}+A_{y}\right) / 2$ - average value of the moment of inertia and $f_{\mathrm{T}}$ - the torsional frequency. Estimated values of moment of inertia $(A)$ and the torsional frequency $\left(f_{\mathrm{T}}\right)$ values are given in Table II and Table III.

TABLE III

Estimated mean square angular amplitudes $\left\langle\theta^{2}\right\rangle$ of the $\mathrm{C}-\mathrm{Cl}$ directions and cor-

\begin{tabular}{|c|c|c|c|c|c|c|}
\hline \multirow{2}{*}{$\begin{array}{l}\text { Sl. } \\
\text { No. }\end{array}$} & \multirow[t]{2}{*}{ Compound } & \multirow[t]{2}{*}{ Atom } & \multirow{2}{*}{$\begin{array}{c}\mathrm{C}-\mathrm{Cl} \\
\text { bond } \\
\text { length } \\
{[\mathrm{pm}]}\end{array}$} & \multirow{2}{*}{$\begin{array}{c}\left\langle\theta^{2}\right\rangle \\
{\left[\operatorname{rad}^{2}\right]}\end{array}$} & \multicolumn{2}{|c|}{$\begin{array}{c}\text { Torsional } \\
\text { frequency }\left(f_{\mathrm{T}}\right)\end{array}$} \\
\hline & & & & & $\begin{array}{l}\text { X-ray } \\
\text { data } \\
{\left[\mathrm{cm}^{-1}\right]}\end{array}$ & $\begin{array}{c}\text { NQR } \\
\text { data } \\
{\left[\mathrm{cm}^{-1}\right]}\end{array}$ \\
\hline \multirow[t]{2}{*}{1} & $\begin{array}{l}\text { 2,5-dichlorophenol } \\
\text { line I }\end{array}$ & $\mathrm{Cl}(1)$ & 172.0 & 0.0080705 & 58.90 & 60 \\
\hline & line II & $\mathrm{Cl}(2)$ & 174.0 & 0.0062486 & 67.00 & 62 \\
\hline \multirow[t]{3}{*}{2} & 2,6-dichlorophenol & & & & & \\
\hline & line $I$ & $\mathrm{Cl}(1)$ & 171.0 & 0.0051312 & 47.13 & 38 \\
\hline & line II & $\mathrm{Cl}(2)$ & 174.0 & 0.0055935 & 44.53 & 40 \\
\hline \multirow[t]{3}{*}{3} & 3,5-dichlorophenol & & & & & \\
\hline & line I & $\mathrm{Cl}(1)$ & 176.6 & 0.002750 & 59.71 & 65 \\
\hline & line II & $\mathrm{Cl}(2)$ & 176.9 & 0.002424 & 63.50 & 66 \\
\hline 4 & $\begin{array}{l}\text { 2,5-dichloro-3,6-dihydroxy } \\
\text { p-benzoquinone }\end{array}$ & $\mathrm{Cl}(1)$ & 171.7 & 0.001610 & 62.45 & 58 \\
\hline \multirow[t]{4}{*}{5} & aniline-2,4,5-trichlorophenol & & & & & \\
\hline & line I & $\mathrm{Cl}(1)$ & 172.9 & 0.0026469 & 34.69 & 33 \\
\hline & line II & $\mathrm{Cl}(2)$ & 174.5 & 0.0013021 & 49.47 & 47 \\
\hline & line III & $\mathrm{Cl}(3)$ & 174.7 & 0.0026010 & 34.54 & 42 \\
\hline 6 & 2-amino-5-chloropyridine & $\mathrm{Cl}(1)$ & 173.6 & 0.0065500 & 44.15 & 42 \\
\hline 7 & monochloro acetic acid & $\mathrm{Cl}(1)$ & 177.0 & 0.0025170 & 22.56 & 20.5 \\
\hline
\end{tabular}
responding effective torsional frequencies $f_{\mathrm{T}}$ at $T=300 \mathrm{~K}$ in the following compounds. 


\section{Results and discussion}

Generally, the torsional frequencies of the molecules lie in the range $20 \mathrm{~cm}^{-1}$ to $100 \mathrm{~cm}^{-1}[5]$. In the present case, the values obtained by X-ray thermal parameters, were compared with those obtained by NQR data [6-8]. It was found that the values obtained in both cases are in good agreement. The above approach is a good illustration of the supplementary nature of the data from X-ray studies [9-12] in relation to NQR studies of compounds in solid state. It is also interesting to compare this value with the Raman data and infrared data.

\section{References}

[1] H. Bayer, Z. Phys. 130, 227 (1951).

[2] M.S. Vijaya, J. Ramakrishna, Mol. Phys. 19, 131 (1970).

[3] T. Kushida, J. Sci. Hiroshima Univ. A 19, 327 (1955).

[4] R.J.C. Brown, J. Chem. Phys. 32, 116 (1960).

[5] H.D. Stidham, Chem. Phys. 49, 2041 (1968).

[6] R. Chandramani, N. Devaraj, V.S. Sastry, J. Ramakrishna, Aust. J. Chem. 29, 2363 (1976).

[7] R. Chandramani, Ph.D. thesis, Bangalore University, India 1977.

[8] S.P. Basavaraj, Ph.D. thesis, Bangalore University, India 1980.

[9] I. Van Bellingen, G. Germain, P. Piret, M. Van Meerssche, Acta Crystallogr. B 27, 553 (1971).

[10] A. Kvick, M. Backeus, Acta Crystallogr. B 30, 474 (1974).

[11] C. Bavoux, P. Michel, Acta Crystallogr. B 30, 2043 (1974).

[12] C. Bavoux, A. Thozet, Acta Crystallogr. B 29, 2603 (1973). 